\title{
Drosophila Pelle phosphorylates Dichaete protein and influences its subcellular distribution in developing oocytes
}

\author{
MOUSUMI MUTSUDDI*,1, ASHIM MUKHERJEE\#,1, BAOHE SHEN",3, JAMES L. MANLEY\#,3 \\ and JOHN R. NAMBU*,2 \\ ${ }^{1}$ Department of Molecular and Human Genetics, Banaras Hindu University, Varanasi-221005, India, \\ ${ }^{2}$ Department of Biology, University of Massachusetts, Amherst, MA, USA and \\ ${ }^{3}$ Department of Biological Sciences, Columbia University, New York, NY, USA
}

\begin{abstract}
The Drosophila Dichaete gene encodes a member of the Sox family of high mobility group (HMG) domain proteins that have crucial gene regulatory functions in diverse developmental processes. The subcellular localization and transcriptional regulatory activities of Sox proteins can be regulated by several post-translational modifications. To identify genes that functionally interact with Dichaete, we undertook a genetic modifier screen based on a Dichaete gain-offunction phenotype in the adult eye. Mutations in several genes, including decapentaplegic, engrailed and pelle, behaved as dominant modifiers of this eye phenotype. Further analysis of pelle mutants revealed that loss of pelle function results in alterations in the distinctive cytoplasmic distribution of Dichaete protein within the developing oocyte, as well as defects in the elaboration of individual egg chambers. The death domain-containing region of the Pelle protein kinase was found to associate with both Dichaete and mouse Sox2 proteins, and Pelle can phosphorylate Dichaete protein in vitro. Overall, these findings reveal that maternal functions of pelle are essential for proper localization of Dichaete protein in the oocyte and normal egg chamber formation. Dichaete appears to be a novel phosphorylation substrate for Pelle and may function in a Pelle-dependent signaling pathway during oogenesis.
\end{abstract}

KEY WORDS: Dichaete, Pelle, oogenesis, phosphorylation

\section{Introduction}

Sox HMG domain proteins act as transcription factors and chromatin architectural proteins and have crucial gene regulatory functions in many developmental processes (reviewed in Guth and Wegner, 2008; Kiefer, 2007). There exist over 20 mammalian Sox genes and several are associated with several human genetic disorders, including sex reversal, campomelic dysplasia, and Waardenburg-Hirschprung disease (reviewed in Chew and Gallo, 2009) and cancer (reviewed in Dong et al. 2004). Thus, illuminating the activities of Sox proteins and how they are regulated is of great significance. Interestingly, several Sox genes act early in cell differentiation pathways and many are highly expressed in progenitor or precursor cell types (Chew and Gallo, 2009); Sox2 has critical functions in regulating pluripotency (reviewed in Zhao and Dailey, 2008). Invertebrate Sox genes also mediate important developmental functions (reviewed in Phochanukul and Russell 2009) and the DrosophilaDichaete Sox gene is essential for embryonic segmentation and nervous system formation (Nambu and Nambu, 1996; Russell et al. 1996). Dichaete exhibits strong sequence similarity to Sox2 and has similar DNA binding and bending activities (Ma et al. 1998). Consistent with functions as a regulator of gene transcription, in most cells Dichaete protein exhibits nuclear localization.

Dichaete also has maternal functions that are important for normal oogenesis and Dichaete protein is expressed transiently and specifically in the developing oocyte (Mukherjee et al. 2006). Interestingly, Dichaete protein exhibits strictly cytoplasmic distri-

Abbreviations used in this paper: HMG, high mobility group; NLS, nuclear localization signal.

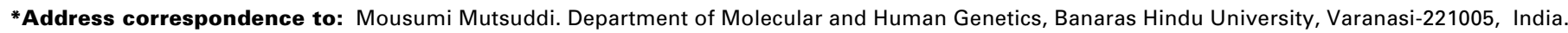

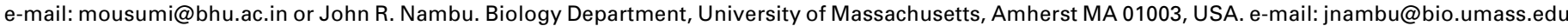

\# Note: These authors contributed equally to this work
} 
bution within the oocyte cytoplasm; it is localized in a crescent at the posterior edge of the oocyte nucleus from region II of the germarium through stage 6 oocytes. Dichaete mutant egg chambers exhibit dorsal/ventral pattern disruptions and Dichaete was shown to have sequence specific RNA-binding properties. Dichaete binds to the 3'-UTR of gurken mRNA and influences distribution of gurken mRNA and protein in the oocyte. Establishment of the anterior/posterior and dorsal/ventral axes during Drosophila oogenesis relies on highly regulated and asymmetric localization of specific mRNAs and proteins (reviewed in Kugler and Lasko 2009). Thus the unique distribution pattern of Dichaete suggests that specific signaling processes may regulate Dichaete localization in the oocyte. Significantly, the mechanisms that underlie this unique localization in the oocyte have not yet been established

In a genetic modifier screen to identify genes that influence Dichaete function, we identified several cell signaling pathway genes, including decapentaplegic, engrailed, and pelle. Mutations in each of these genes exhibited dominant modification of a gain-of-function Dichaete adult eye phenotype. We focused further attention on pelle, which encodes a conserved serine/threonine protein kinase that also contains a death domain and acts as a downstream transducer in the Toll signaling pathway (Shelton and Wasserman, 1993). Significantly, the Pelle death domain mediates direct association with other proteins, such as Tube (Xiao et al. 1999), and Pelle kinase activity influences the stability and subcellular localization of members of the Dorsal/Nf-kB transcription factor family (Shen and Manley, 1998; Towb et al. 1998; Edwards et al. 1997). In addition to it's critical maternal functions in dorsal/ventral patterning of the embryo Pelle also has important zygotic functions in hematopoesis, muscle develop- ment, axon guidance and innate immune response (Mindorff et al. 2007; Halfon and Keshishian, 1998; Qu et al. 1998; Lemaitre et al. 1996). Identifying additional Pelle interacting or substrate proteins is thus relevant for a wide array of developmental and physiological processes. In this study, we characterize functional interactions between Pelle and Dichaete. We show that maternal Pelle functions are required for the normal distribution of Dichaete protein in the developing oocyte, and that pelle mutant ovaries exhibit defects in the elaboration of individual egg chambers. The death domain-containing region of Pelle strongly associates with Dichaete as well as the mouse Sox 2 protein. In addition, in vitro kinase assays indicated that Pelle can phosphorylate Dichaete. Taken together, our results suggest that phosphorylation of Dichaete by Pelle may be important for normal oogenesis.

\section{Results}

\section{A genetic modifier screen identifies enhancers of Dichaete function}

A genetic modifier screen was performed to identify genes that influence Dichaete function. P[GMR-ga/4] was used to target ectopic expression of Dichaete in the developing eye imaginal disc via P[UAS-Dichaete]. Flies bearing these two $P$ elements exhibit a dosage-sensitive loss and disorganization of ommatidia and mechanosensory bristles in the adult eye (Mukherjee et al. 2000). P[GMR-ga/4]/CyO; P[UAS-Dichaete]/TM3 flies were crossed to a series of 169 autosomal deficiencies that span most of the second and third chromosomes and the progeny were scored for alterations in the adult eye phenotype. The screen identified 11 regions on the second chromosome (21D1-2;22B2-
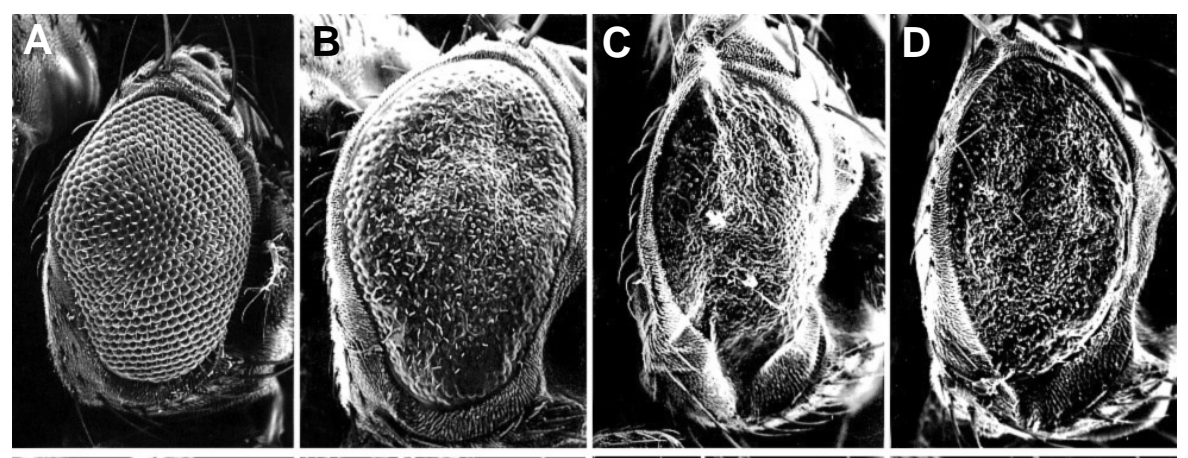

Fig. 1. Dominant modification of a Dichaete gainof-function eye phenotype by chromosomal deficiencies and specific gene mutations. (A) The head capsule of a P[GMR-gal4]/+ fly. Note the ordered array of ommatidia and mechanosensory bristles in the compound eye. (B) The head capsule of a P[GMR-gal4]/+; PIUAS-Dichaete $] /+$ fly. Note the loss and disorganization of the ommatidia and mechanosensory bristles. (C) Heterozygosity for Df(2L)C144 (23A1-2; 23C3-5) enhances theÄDichaete gain-of-function eye phenotype. Note the more reduced and collapsed eye with severe loss of mechanosensory bristles in a P[GMR-gal4]/
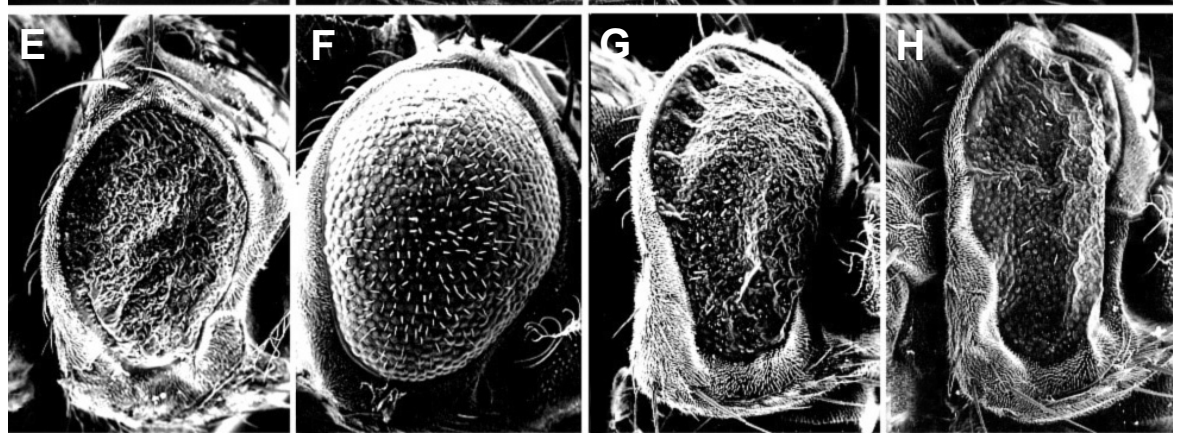
Df(2L)C144; PIUAS-Dichaete]/+ fly. (D) Heterozygosity for Df(2R)en-A (47D3; 48B2-5) which removes engrailed, enhances the Dichaete gain-of-function eye phenotype. Note the more severe loss of mechanosensory bristles in a P[GMR-gal4]/Df(2R)enA; P[UAS-Dichaete]/+ fly. (E) Heterozygosity for Df(2L)H20 (36A8-9; 36E1-2) enhances the Dichaete gain-of-function eye phenotype. Note the more reduced size of the eye and greater disruption of ommatidial organization in a P[GMR-gal4]/Df(2L)H2O; P[UAS-Dichaete]/+ fly. (F) Heterozygosity for Df(3R)P14 (90C2-D1; 91A1-2) which removes glass, suppresses the Dichaete gain-of-function eye phenotype. Note the rescue of eye size and ommatidial organization in a P[GMR-gal4]/+; PIUAS-Dichaete]/Df(3R)P14 fly. (G) Heterozygosity for Df(3R)R38.3 (97E3-11; 98A), which removes pelle, enhances the Dichaete gain-of-function eye phenotype. Note the collapsed eye with severe loss of mechanosensory bristles in a PIGMR-gal4]/+; PIUAS-Dichaete]/Df(3R)R38.3 fly. (H) Heterozygosity for Df(3R)IR16 (97F1-2; 98A), which removes pelle, enhances the Dichaete gain-of-function eye phenotype. Note the more reduced and collapsed eye with severe loss in mechanosensory bristles in a PIGMR-gal4]/+; PIUAS-Dichaete]/Df(3R)IR16 fly. (A-H) Scanning electron micrographs of 2-3 day old adult flies at 200X magnification. 

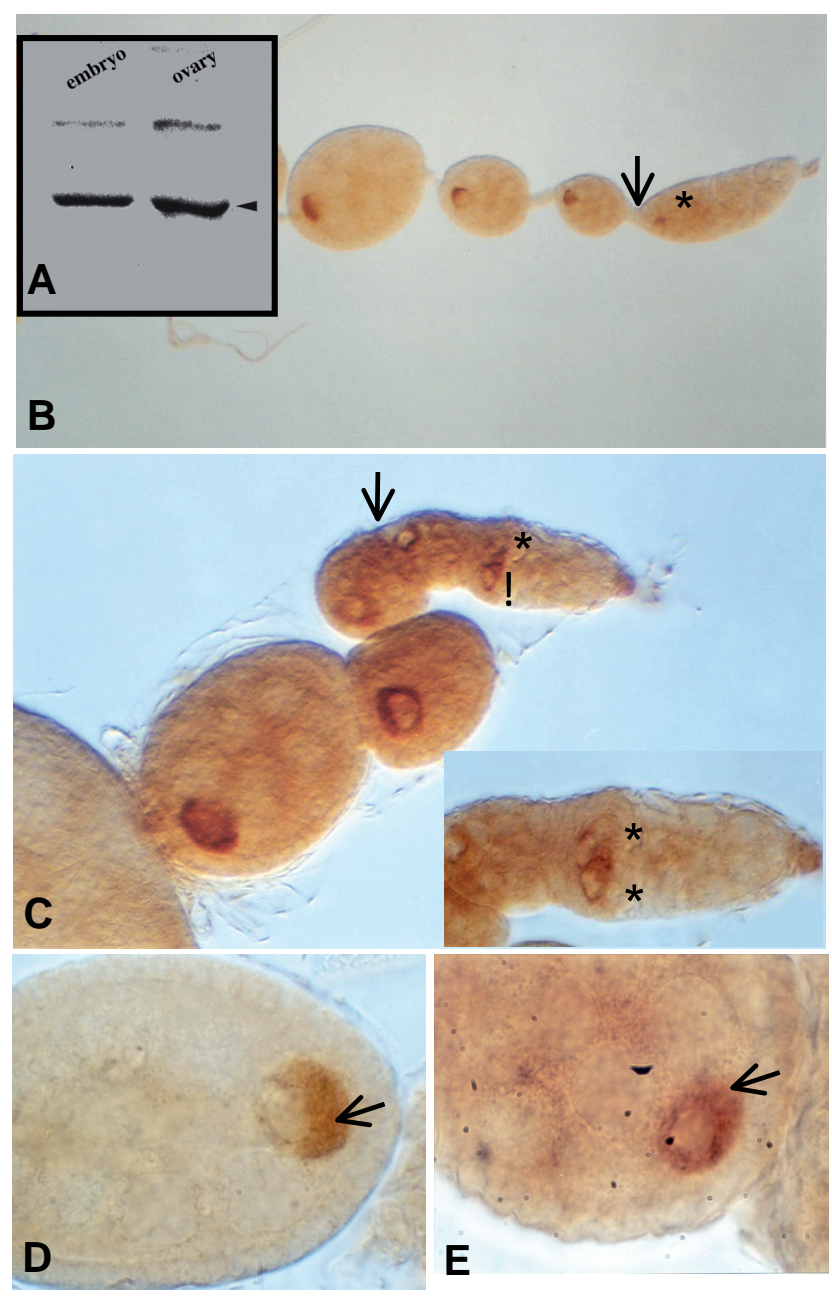

Fig. 2. pelle is expressed ovaries and is required for proper distribution of Dichaete protein in the oocyte as well as egg chamber separation. (A) Pelle protein expression was detected in 0-3 hour wild type embryos and dissected adult female ovaries (arrowhead) via Western blot analysis. (B-E) Anti-Dichaete immunostaining was performed on ovaries dissected from wild type $(B, C)$ and pelle mutant $(D, E)$ females. (B) In wild type ovarioles, the germarium (arrow) is separated from the stage 1 oocyte by somatic stalk cells. Note the presence of a single cell expressing Dichaete in the germarium (*) and stage 1 oocyte. (C) In an ovariole from a pelle ${ }^{\mathrm{rm} 8} / \mathrm{Df}(3 R)$ 38.3. mutant female the germarium (arrow) and stage 1 oocyte are fused, and the stalk is absent. Note also that Dichaete protein is detected around two distinct nuclei in the germarium $(*)$. Inset showing a higher magnification. (D) In a stage 5 wild type oocyte Dichaete protein is specifically localized around the posterior of the oocyte nucleus (arrow). (E) In a stage 5 oocyte derived from a pelle rm8/ Df(3R)38.3 mutant female, Dichaete protein is not restricted to the posterior cytoplasm and is dispersed around the entire oocyte nucleus (arrow; also see panel B).

3, 22F;23D1-2, 24C2;25D3-4, 35E1-2;36A, 36C2-4;37B9, 38A06B01;40A, 41D;42B1-3, 42C1;43E, 45D9;46F1-2, 47D3;48C6-8, and $54 \mathrm{EF} ; 55 \mathrm{~B} 9-\mathrm{C} 1)$ and 2 regions on the third chromosome $(63 \mathrm{C} 1 ; 63 \mathrm{D} 3,90 \mathrm{~F} 1-4 ; 91 \mathrm{~A} 1-2$ and $97 \mathrm{~A} ; 98 \mathrm{~A} 5)$ that behaved as genetic enhancers of the Dichaete induced eye phenotype. These enhancers all resulted in more severe disorganization of eye tissue (Fig. 1 A-E,G,H). Three suppressors were also identified that all removed regions containing the glass gene (Fig. 2F) and presumably result in reduced Glass-dependent ga/4and Dichaete expression.

To identify the specific modifier genes, single gene mutations that mapped within the relevant deficiency intervals were crossed to P[GMR-ga/4]/CyO; P[UAS-Dichaete]/TM3 flies. This analysis revealed that mutations in decapentaplegic $\left(d p p^{S 1}\right.$ and $d p p^{d-h o}$ alleles), engrailed ( $e n^{1}$ allele), and pelle ( $\mathrm{Df}(3 \mathrm{R}) R 38.3$ allele) mimicked the effects of the corresponding deficiencies at $22 \mathrm{~F}$, $48 \mathrm{~A}$, and $97 \mathrm{~F}$, respectively (Fig. $2 \mathrm{G}, \mathrm{H}$ ). $d p p$ encodes a TGF- $\beta$ related protein that plays an essential role in morphogenetic furrow formation and retinal patterning during eye development (Cordero et al. 2007). Based on the ability of ectopic Dichaete to repress $d p p$ expression in the eye disc (Mukherjee et al. 2000), removal of one copy of $d p p$ could exacerbate the P[GMR-ga/4]/ $\mathrm{P}[\mathrm{UAS}$-Dichaete] eye phenotype by further reducing levels of $d p p$ expression. The en gene encodes a conserved homeodomain protein whose precise role in eye development is unclear. Loss of Dichaete does disrupt en expression in the embryo and larval CNS (Mukherjee et al. 2000; Nambu and Nambu, 1996; Russell et al. 1996) suggesting ectopic Dichaete could influence en expression in the eye imaginal disc. Alternately, En could repress the activities of Dichaete in the eye disc; direct interactions between other homeodomain and Sox proteins have been reported (Wissmüller et al. 2006). The ability of pelle mutants to modify the Dichaete gain-of-function eye phenotype suggests that pelle may normally function in eye development. pelle mutants do exhibit mis-targeting of photoreceptor R1-5 axons to the medulla (Mindorff et al. 2007), suggesting that pelle may attenuate the effects of ectopic Dichaete expression in the developing eye disc. As there is only limited knowledge of the signaling pathways that influence Sox protein activities, we chose to further investigate functional interactions between Dichaete and Pelle.

\section{Pelle is required for proper distribution of Dichaete protein in the oocyte and egg chamber separation}

Maternal pel/efunction is required for embryonic dorsal/ventral pattern formation and Western blot analyses confirmed that Pelle protein is expressed in early embryos as well as adult female ovaries (Fig. 2A). Dichaete is also expressed during oogenesis (Mukherjee et al. 2006), and strikingly, in early stage egg chambers Dichaete protein is specifically restricted to the cytoplasm around the posterior margin of the oocyte nucleus (Fig. 2B). We thus examined whether pelle might influence Dichaete expression or distribution by performing anti-Dichaete immunostaining on ovaries from pelle ${ }^{385} \mathrm{Df}(3 \mathrm{R}) 38.3$ and pel/e ${ }^{\mathrm{rm} 8} \mathrm{Df}(3 \mathrm{R}) 38.3$ mutant females. This analysis indicated that Dichaete protein is still expressed and present in the cytoplasm of pelle mutant oocytes. However, in $12.8 \%$ (6 out of 47 ) of stage 5-6 pelle ${ }^{385}$ / $\mathrm{Df}(3 \mathrm{R}) 38.3 \mathrm{and} 20.5 \%$ (9 out of 44 ) of stage $5-6$ pelle $\mathrm{e}^{\mathrm{rm}} \mathrm{Df}(3 \mathrm{R}) 38.3$ mutant oocytes, Dichaete protein exhibited an abnormal diffuse distribution and was not restricted to the posterior cytoplasm (Fig. $2 \mathrm{C}$ ). In addition, pellemutant ovaries also exhibited defects in the elaboration of individual egg chambers. Unlike wild type ovarioles, $12.8 \%$ (6 out of 47 ) of the pel/e $385 \mathrm{Df}(3 \mathrm{R}) 38.3$ and $6.8 \%$ (3 out of 44) of the pel/erms $\mathrm{Df}(3 \mathrm{R}) 38.3$ ovarioles contained two or more fused egg chambers (Fig. 2 D,E). In addition, the stalk cells between adjacent egg chambers were often lacking and some mutant egg chambers contained multiple sites of Dichaete ex- 

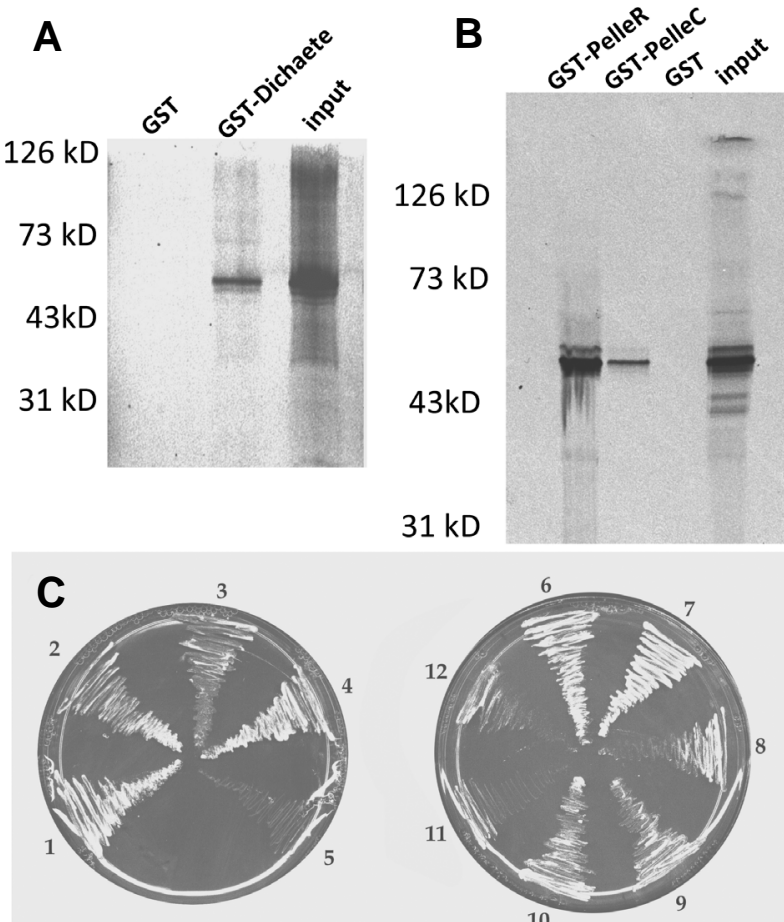

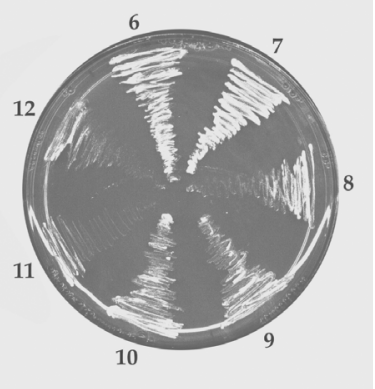

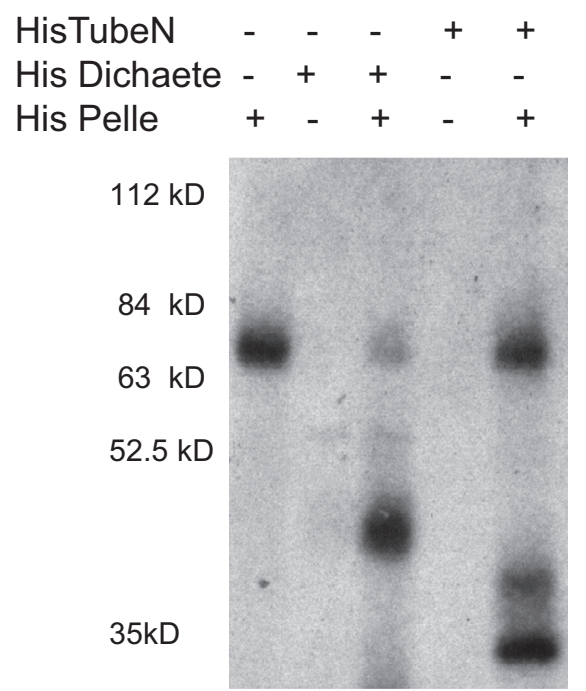

Fig. 3 (Left). Dichaete associates with the death domain-containing region of Pelle. (A,B) GST-pulldown assays using Dichaete and Pelle proteins. (A) Binding of a GST-Dichaete fusion protein to ${ }^{35} \mathrm{~S}$-labeled full length Pelle generated via in vitro translation. ${ }^{35} \mathrm{~S}$-labeled Pelle protein migrates at $\sim 55 \mathrm{kD}$ (input). Pelle protein specifically associated with GSTDichaete and not with GST alone. (B) Binding of GST-Pelle proteins to ${ }^{35} \mathrm{~S}$ labeled full length Dichaete generated via in vitro translation. ${ }^{35}$ S-labeled full length Dichaete migrates at $\sim 45 \mathrm{kD}$ (input). Dichaete protein associ-

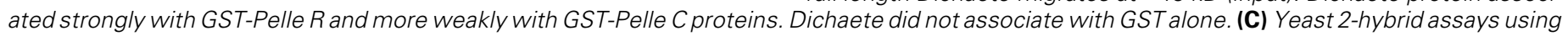

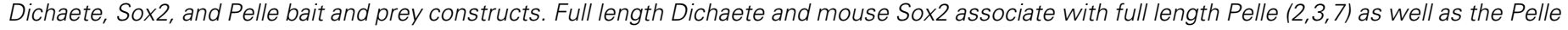

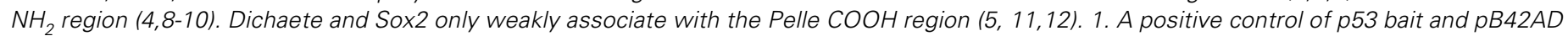

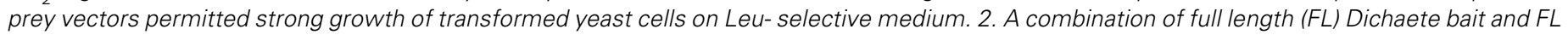

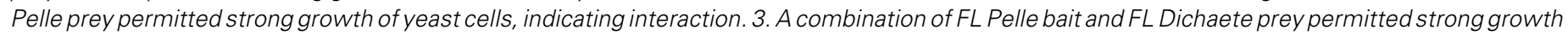

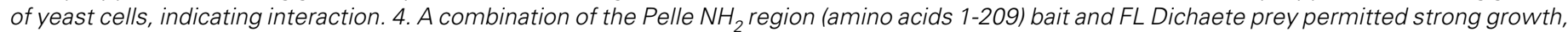

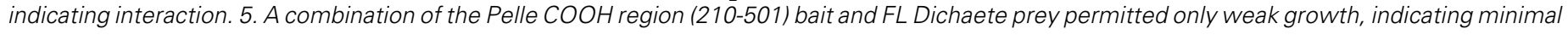

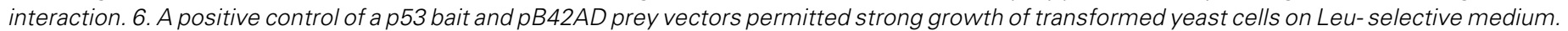

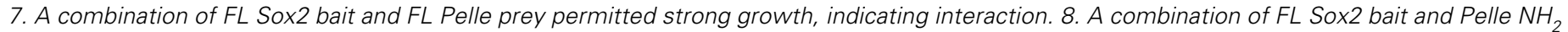

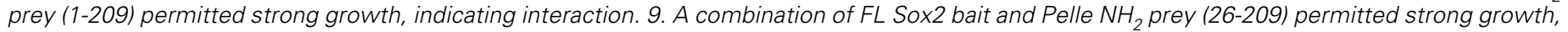

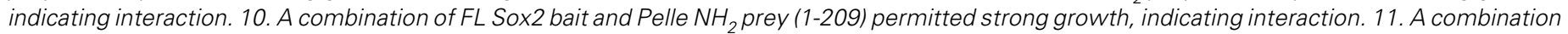

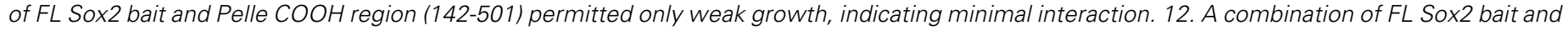
Pelle COOH region (210-501) permitted only weak growth, indicating minimal interaction.

Fig. 4 (Right). Pelle phosphorylates Dichaete protein. Autoradiogram from an in vitro kinase assay using purified 6XHis-Pelle, 6XHis-Dichaete, and 6 XHis-TubeN proteins. Note that Pelle exhibits autophosphorylation activity as evidenced by a prominent ${ }^{32} \mathrm{P}$-labeled band at $\sim 65 \mathrm{kD}$ [see Shen and Manley, 2002]. Pelle also phosphorylates the $\mathrm{NH}_{2}$ region of Tube (note bands at $\sim 35 \mathrm{kD}$ ) and Dichaete (note band at $\sim 48 \mathrm{kD}$ ). No labeling of Dichaete or TubeN proteins were detected in the absence of Pelle protein.

pression. Thus, maternal pelle functions are important both for proper distribution of Dichaete protein in the oocyte and normal elaboration of individual egg chambers.

\section{Pelle directly associates with and phorphorylates Dichaete}

To determine whether the Pelle and Dichaete proteins may directly interact, GST-pulldown and yeast 2-hybrid assays were performed. ${ }^{35}$ S-labeled full length Pelle protein specifically associated with GST-Dichaete, but not GST alone (Fig. 3A). In addition, ${ }^{35}$ S-labeled full length Dichatete associated strongly with GSTPelle R (amino acids 1 to 209 of Pelle) which includes the death domain and weakly with GST-Pelle C (amino acids 209 to 501 of Pelle) which includes the kinase catalytic domain (Fig. 3B). Yeast 2-hybrid assays also indicated association between full length Dichaete and Pelle proteins (Fig. 3C) and further revealed that Dichaete strongly associated with the Pelle $\mathrm{NH}_{2}$ region (1-209) and only weakly associated with the Pelle $\mathrm{COOH}$ region (amino acids 210-501). These data indicate that Dichaete and Pelle proteins can directly associate and that these interactions are predominantly mediated via the death domain-containing $\mathrm{NH}_{2}$ region of Pelle. As the HMG domain of Dichaete is $88 \%$ identical to that of Sox2, additional yeast 2-hybrid assays were carried out to show that full length Pelle can also associate with Sox2 (Fig. 3C). Similar to Dichaete, Sox2 exhibited strong interaction with the Pelle $\mathrm{NH}_{2}$ region (amino acids 1-209 or 26-209) and a weak interaction with the Pelle $\mathrm{COOH}$ region (amino acids $142-501$ or $210-501$ ). This result suggests that the HMG domain of Dichaete and Sox2 is important for association with Pelle.

The ability of Dichaete and Pelle proteins to directly associate suggested that Dichaete might be a phosphorylation substrate for Pelle. To test this hypothesis, in vitro kinase assays were performed using full length 6XHis-Pelle and 6xHis-Dichaete proteins. 
As previously demonstrated (Shen and Manley, 2002; Shen and Manley, 1998), incubation of 6xHis-Pelle in the presence of $\gamma^{-32} \mathrm{P}$ ATP results in autophosphorylation, and 6xHis-Pelle also phosphorylates the $\mathrm{NH}_{2}$ region of Drosophila Tube (Fig. 4). Co-incubation of 6xHis-Pelle and 6xHis-Dichaete resulted in the presence of a prominent ${ }^{32} \mathrm{P}$-labeled band corresponding to $6 x \mathrm{His}$-Dichaete (Fig. 4). These data thus identify Dichaete as a novel target for the Pelle kinase.

\section{Discussion}

\section{Regulation of Sox protein functions by post-translational modifications}

Sox proteins can undergo several types of post-translational modification that influence their subcellular localization and transcriptional activation properties (reviewed in Wegner, 2005). Consistent with their functions as transcription regulators, Sox proteins are typically detected in cell nuclei and the HMG domains of several Sox proteins, including Dichaete, contain a bipartite and a basic cluster nuclear localization signal (NLS) (reviewed in Smith and Koopman, 2004). The absence of Dichaete protein in the oocyte nucleus suggests that these NLSs are masked, either via a distinct Dichaete protein conformation, binding to another molecule, or a post-translational modification. Several Sox proteins have been shown to undergo phosphorylation, including Sry, Sox9, and Sox3 (Huang et al. 2000; Desclozeaux et al. 1998; Stukenberg et al., 1997). While the site of Pelle-mediated Dichaete phosphorylation is not yet established, use of the NetPhos 2.0 Protein Phosphorylation Prediction Server (http://www.cbs.dtu.dk/services/NetPhos/ ) identified 17 consensus phosphorylation sites in Dichaete (data not shown). Two of the predicted sites reside within the HMG domain; Ser186 (context = LLAESEEKRP) received a score of 0.996 (maximum score $=1.0$; scores over 0.5 are predictive) and a Tyr209 (context = EHPDYKYRP) received a score of 0.942. Similar potential phosphorylation sites were predicted in the HMG domain of Sox2 (data not shown). The Dichaete basic cluster NLS overlaps the predicted Tyrosine phosphorylation site, suggesting that phosphorylation at this site might influence the activity of this NLS. However, Protein Kinase A-mediated phosphorylation of Serine211 near the basic cluster NLS of Sox9 did not alter its nuclear localization (Huang et al. 2000).

While Pelle kinase does not appear to be responsible for controlling cytoplasmic versus nuclear localization of Dichate in the oocyte, its absence does result in more diffuse localization of Dichaete protein. These findings suggest that Pelle-mediated phosphorylation is important for proper positioning of Dichaete in the oocyte cytoplasm and that distinct post-translational mechanisms regulate it's cytoplasmic versus nuclear distribution. Acetylation of both Sry and Sox2 regulates nuclear localization (Baltus et al. 2009) and this acetylation is mediated by the p300 histone acetyl transferase (HAT) at the lysine residue in an ISKRL motif in the central portion of the HMG domain. This motif and the lysine residue are strongly conserved among vertebrate Sox2 proteins and are also present in the Dichaete HMG domain. Perhaps constitutive modification of this site by a DrosophilaHAT maintains cytoplasmic localization of Dichaete in the oocyte.

\section{Functions for Dichaete and Pelle in oogenesis}

This study revealed functions of pelle in oogenesis; pelle
mRNA expression was detected in dissected ovaries and pelle mutants exhibited a partially penetrant defect in egg chamber separation. In addition, multiple sites of Dichaete expression were detected in some pelle mutant egg chambers. It is possible that pelle is required for the normal function of stalk cells and other follicle cells in the individualization of adjacent egg chambers. This phenotype resembles that seen for germline mutations in the gene encoding a nonmotor kinesin II-associated protein (Pflanz et al. 2004). The ectopic sites of Dichate expression could correspond to potential oocyte duplication or failure of developing cysts to fully separate. While the important sites of pelle expression during oogenesis are unknown, pelle mutants do appear to affect relatively early stages of oogenesis as defects in egg chamber separation and Dichaete expression were observed in the germarium. As yet there is little evidence indicating a role for Sox proteins in the Toll/Interleukin-1 Receptor signaling pathway. However, Pelle can function in distinct pathways (Murdiff et al. 2007) and Pelle may influence Dichaete distribution independently of the Toll pathway. Taken together, the data suggest that a non-canonical Pelle pathway may influence Dichaete localization during oogenesis. Given the tightly regulated localization and translation of mRNAs in the Drosophila oocyte (reviewed in Kugler and Lasko 2009), the highly restricted positioning of Dichaete protein could be important for post-transcriptional regulatory processes during oogenesis.

\section{Materials and Methods}

\section{Drosophila stocks}

Generation of P[UAS-Dichaete] is described in (Mukherjee et al. 2000). P[GMR-ga/4] and the second and third chromosome deficiency kits were obtained from the Bloomington Drosophila Stock Center. $\mathrm{Df}(3 \mathrm{R}) \mathrm{R} 38.3$ (97E3-11; 98A) is a $\mathrm{P}$ element excision pelle null allele (Hecht and Anderson, 1993), Df(3R)IR16 (97F1-2; 98A) is a $\gamma$-ray induced null allele (Shelton and Wasserman 1993), and $p / \beta^{85}$ and $p / /^{m 8}$ are EMSinduced strong alleles (Hecht and Anderson, 1993).

\section{Genetic modifier screen}

P[GMR-ga/4]/CyO; P[UAS-Dichaete]/TM3 females were crossed to males carrying a second or third chromosome deficiency carried over a balancer chromosome. At least 100 non-balancer progeny from each cross were analyzed for adult eye morphology and the phenotypes were compared to P[GMR-ga/4]/+; P[UAS-Dichaete]/+ flies. Modifier phenotypes were confirmed via secondary crosses between P[GMR-ga/4]/CyO; P[UAS-Dichaete]/TM3 males and deficiency chromosome females. All crosses were maintained at $25^{\circ} \mathrm{C}$.

\section{Scanning electron microscopy}

Two to three day old adult flies were prepared for scanning electron microscopy by fixation with $2 \%$ glutaraldehyde in PBS $(175 \mathrm{mM} \mathrm{NaCl}$, $1.86 \mathrm{mM} \mathrm{NaH}_{2} \mathrm{PO}_{4} 8.41 \mathrm{mM} \mathrm{Na}_{2} \mathrm{HPO}_{4} \mathrm{pH}$ ) for 6 hours on ice, followed by dehydration through a graded ethanol series. Samples were critical point dried in a Polaron CPD unit and were sputter coated with palladium/gold. They were examined and photographed using the UMass Central Microscopy Facility (http://www.bio.umass.edu/microscopy/) using a Nikon JEOL 5400 scanning electron microscope.

\section{Immunostaining and immunoblotting}

Anti-Dichaete immunostaining of ovarioles was performed using antiDichaete serum (Ma et al. 1998) at a 1:1000 dilution in PBT (PBS and $0.1 \%$ Triton $\mathrm{X}-100$ ) and a biotin-conjugated anti-rabbit secondary antibody (Vector Laboratories) at a 1:400 dilution. Detection of antibody 
labeling was achieved using streptavidin-horseradish peroxidase (Vector Laboratories) and diaminobenzidine $/ \mathrm{H}_{2} \mathrm{O}_{2}$ reactions.

For Western blot analysis, protein extracts were prepared from $100 \mu \mathrm{l}$ of dechorionated 0-3 hour Canton-S embryos or $\sim 50$ ovaries dissected from adult female flies. Tissues were homogenized in $100 \mu$ l of lysis buffer provided in the Immunoprecipitation Kit (Boehringer Mannheim) and centrifuged to pellet debris. $20 \mu$ l of protein extracts were electrophoresed on an $8 \%$ SDS polyacrylamide gel and electro-transferred to nitrocellulose membrane. Anti-Pelle serum was purified by preabsorption to fixed third instar larval salivary glands (lacking associated fat body) for one hour. The membranes were blocked in PBS containing 5\% nonfat dry milk and $0.2 \%$ Tween 20 for one hour, and then incubated with a 1:1000 dilution of preabsorbed anti-Pelle serum at $4^{\circ} \mathrm{C}$ overnight. The blot was washed in PBS $+0.2 \%$ Tween 20 , incubated in biotin-conjugated antirabbit secondary antibody (Vector Laboratories) for one hour at room temperature, and washed several times. Labeled proteins were detected via chemiluminescence using reagents from the ECL kit (Amersham Lifesciences) and autoradiography.

\section{GST-pulldown assays}

Preparation of GST-Dichaete fusion protein using a pGEX-2T subclone was performed as described in (Ma et al. 2000). GST-Pelle R and GSTPelle $\mathrm{C}$ fusion proteins were prepared as described in (Shen and Manley, 1998). ${ }^{35}$ S-labeled full length Dichaete or Pelle proteins were generated using full length Dichaeteand pellecDNA clones via in vitrotranscription/ translation reactions (Promega) and ${ }^{35} \mathrm{~S}$-methionine (Amersham Pharmacia). GST-pulldown assays were performed by incubating 1-4 $\mu \mathrm{g}$ of purified GST fusion protein (GST-Dichaete, GST-Pelle R, or GST-Pelle C) with $20 \mu$ l of glutathione-sepharose-4B beads in $100 \mu$ l of NETN $(20 \mathrm{~mm}$ Tris- $\mathrm{HCl} \mathrm{pH} \mathrm{8.0,100mm} \mathrm{NaCl,} 1 \mathrm{~mm}$ EDTA and 0.5\% NP40) for 2 hours at $4{ }^{\circ} \mathrm{C}$ followed by several washes with NETN. $5 \mu$ of in vitro translated ${ }^{35} \mathrm{~S}$-methionine-labelled protein (Dichaete or Pelle) was incubated with 20 $\mu$ l of glutathione-sepharose-4B beads in $40 \mu \mathrm{l}$ of NETN for 2 hours at $4{ }^{\circ} \mathrm{C}$. The supernatant containing the ${ }^{35} \mathrm{~S}$-methionine-labelled protein was then transferred to the sepharose-4B beads bound to GST-Dichaete or GSTPelle and incubated for 2-6 hours at $4{ }^{\circ} \mathrm{C}$. The complexes were washed several times with NETN and bound protein eluted with buffer containing reduced glutathione (Amersham Pharmacia). The eluted samples were briefly boiled and electrophoresed on either an $8 \%$ or $10 \%$ SDS polyacrylamide gel. The gels were fixed in 1:1 methanol:acetic acid, dried, and autoradiographed.

\section{Yeast 2-hybrid assays}

The pEG202 bait (fusion to LexA DNA binding domain) and the pJG45 prey (fusion to $B 42$ transcriptional activation domain) vectors (Origene) were used to generate constructs for yeast two hybrid analyses. Full length pel/ebait and prey constructs were generated via PCR using a full length pellecDNA clone (Shelton and Wasserman, 1993) as template and the following oligonucleotide primers:

5'-GAGGCCGAATTCGGCGTCCAGACCGCCGAAGCC-3'

\section{5'-CTTGGCAAACAATGGCTGGAGCTCCGACCG-3'}

The PCR products were digested with EcoRI and Xhol, purified and subcloned into pEG202 and pJG4-5. pEG202 bait constructs expressing Pelle- $\mathrm{NH}_{2}$ (amino acids 1-209) and Pelle-COOH (amino acids 210-501) were kindly provided by Steve Wasserman (Shelton and Wasserman, 1993). Generation of the Dichaete bait and prey constructs are described in (Ma et al. 1998; 2000).

A pEG202 bait construct that expresses full length mouse Sox2 (amino acids 4-319), including the HMG domain, was generated via PCR from a Sox-2 cDNA clone kindly provided by Lisa Dailey. The following primers were utilized:

5'-GGGGGAATTCATGGAGACGGAGCTGAAGCCGCCG-3'

5'-GGGGCTCGAGCATGTGCGACAGGGGCAGTGTGCC-3'

The PCR product was digested with EcoRI and Xhol and subcloned into the pEG202 vector.
Combinations of bait and prey constructs were co-transformed into EGY48 host yeast cells (Mat $\alpha$ trp1 his3 ura3 leu2:::6lexAop-LEU2) according to the supplier's instructions (Origene). The transformed cells were plated on YNB/Leu/Ura (-His, -Trp) glucose medium and incubated at $30^{\circ} \mathrm{C}$ for $3-4$ days. The transformants were then re-plated on the YNB/ Ura (-His, -Leu, -Trp) galactose/raffinose medium and incubated at $30^{\circ} \mathrm{C}$ for 4 days to assay for the presence of colonies and activation of the Leu2 reporter gene.

\section{In vitro phosphorylation assay}

Phosphorylation assays were performed essentially as described in (Shen and Manley, 1998). Reactions with various combinations of 6 xHisPelle, 6xHis- $\mathrm{TubeNH}_{2}$ (amino acids 1 to 259), and 6xHis-Dichaete were incubated for 30 minutes at $30^{\circ} \mathrm{C}$ in $40 \mu$ reactions containing kinase buffer (25mM HEPES pH 7.5, $10 \mathrm{mM} \mathrm{MgCl}_{2}, 5 \mathrm{mM} \mathrm{MnCl}_{2}, 50 \mathrm{mM} \beta$ glycerol phosphate, $20 \mathrm{mM}$ ATP and $5 \mu \mathrm{Ci} \gamma^{32}$ P-ATP). $50 \%$ of each reaction was electrophoresed on an $8 \%$ SDS polyacrylamide gel. The gel was subjected to Coomassie blue staining and autoradiography.

\section{Acknowledgements}

We are indebted to Steve Wasserman for providing us with a pelle cDNA clone, pelle mutant stocks, and pelle yeast 2-hybrid constructs. We thank Lisa Dailey for providing us with a mouse Sox 2 CDNA clone and Yue Ma for assistance generating the Sox2 yeast 2-hybrid construct. We also thank Katthy Matthews, Emma Rushton, Graham Thomas, Kathryn Anderson, and Andreas Bergmann for providing us with fly stocks, and Frank Schnorrer for providing anti-Pelle sera. The scanning electron microscopy was performed by Lucy Yin at the University of Massachusetts Central Microscopy Facility. This work was supported by a March of Dimes Basic Research Grant and NIH grant NS32251 to JRN, NIH grant GM 37971 to JLM, and funds from the Department of Science and Technology, Government of India to MM and AM.

\section{References}

BALTUS, GA., KOWALSKI, MP., ZHAI, H., TUTTER, AV., QUINN, D., WALL, D., KADAM, S. (2009). Acetylation of Sox2 induces its nuclear export in embryonic stem cells. Stem Cells 27: 2175-2184.

CHEW, LJ., AND GALLO, V. (2009). The Ying and Yang of Sox proteins: Activation and repression in development and disease. JNeurosci Res 87: 3277-3287.

CORDERO, JB., LARSON, DE., CRAIG, CR., HAYS, R., CAGAN, R. (2007). Dynamic decapentaplegic signaling regulates patterning and adhesion in the Drosophila pupal retina. Development 134: 1861-1871.

DESClOZEAUX, M., POULAT, F., DE SANTA BARBARA, P., CAPONY, JP., TUROWSKI, P., JAY, P., MEJEAN, C., MONIOT, B., BOIZET, B., BERTA, P. (1998). Phosphorylation of an N-terminal motif enhances DNA-binding activity of the human SRY protein. J Biol Chem 273: 7988-7995.

DONG, C., WILHELM, D., KOOPMAN, P. (2004). Sox genes and cancer. Cytogenet Genome Res. 105: 442-447.

EDWARDS, DN., TOWB., P., WASSERMAN, SA. (1997). An activity-dependent network of interactions links the Rel protein Dorsal with its cytoplasmic regulators. Development 124: 3855-3864.

GUTH, SI., WEGNER, M. (2008). Having it both ways: Sox protein function between conservation and innovation. Cell Mol Life Sci65: 3000-3018.

HALFON, MS., KESHISHIAN, H. (1998). The Toll pathway is required in the epidermis for muscle development in the Drosophilaembryo. Dev Bio/199: 164174.

HECHT, PM., ANDERSON, KV. (1993). Genetic characterization of tube and pelle, genes required for signaling between Toll and dorsal in the specification of the dorsal-ventral pattern of the Drosophila embryo. Genetics 135: 405-417.

HUANG, W., ZHOU, X., LEFEBVRE, V., DE CROMBRUGGHE, B. (2000). Phosphorylation of SOX9 by cyclic AMP-dependent protein kinase A enhances SOX9's ability to transactivate a Col2a1 chondrocyte-specific enhancer. Mol Cell Bio/20: 4149-4158.

KIEFER, JC. (2007). Back to basics: Sox genes. Dev Dyn 236: 2356-2366. 
KUGLER, JM., LASKO, P. (2009). Localization, anchoring, and translational control of oskar, gurken, bicoid, and nanos mRNA during Drosophila oogenesis. Fly (Austin). 3: 15-28.

LEMAITRE, B., NICOLAS, E., MICHAUT, L., REICHHART, JM., HOFFMANN, JA. (1996). The dorsoventral regulatory gene cassette spatzle/Toll/cactus controls the potent antifungal response in Drosophila adults. Ce//86: 973-983.

MA, Y., NIEMITZ, EL., NAMBU, PA., SHAN, X., SACKERSON, C., FUJIOKA, M., GOTO, T., NAMBU, JR. (1998). Gene regulatory functions of Drosophila Dichaete, a high mobility group domain Sox protein. Mech Dev73: 169-182.

MA, Y., CERTEL, K., GAO, Y., NIEMITZ, E., MOSHER, J., MUKHERJEE, A., MUTSUDDI, M., HUSEINOVIC, N., CREWS, ST., JOHNSON, WA., NAMBU, JR. (2000). Functional interactions between Drosophila bHLH/PAS, Sox, and POU transcription factors regulate CNS midline expression of the slit gene. $J$ Neurosci20: 4596-4605.

MINDORFF, EN., O'KEEFE, DD., LABBÉ, A., YANG, JP., OU, Y., YOSHIKAWA, S. VAN MEYEL, DJ. (2007). A gain-of-function screen for genes that influence axon guidance identifies the NF-kappaB protein dorsal and reveals a requirement for the kinase Pelle in Drosophilaphotoreceptor axon targeting. Genetics 2247-2263.

MUKHERJEE, A., SHAN, X., MUTSUDDI, M., MA, Y., NAMBU, JR. (2000). The Drosophila Sox gene, Dichaete, is required for postembryonic development. Dev Bio/217: 91-106.

MUKHERJEE, A., MELNATTUR, KV., ZHANG, M., NAMBU, JR. (2006). Maternal expression and function of the Drosophila sox gene Dichaete during oogenesis. Dev Dyn 10: 2828-2835.

NAMBU, PA., NAMBU, JR. (1996). The Drosophila Dichaete gene encodes a HMG domain protein essential for segmentation and CNS development. Development 122: 3467-3475.

OHE, K., LALLI, E., SASSONE-CORSI, P. (2002). A direct role of Sry and SOX proteins in pre-mRNA splicing. Proc. Natl. Acad. Sci. USA 99: 1146-1151.

PHOCHANUKUL, N., RUSSELL, S. (2009). No backbone but lots of Sox: invertebrate Sox genes. Int J Biochem Cel/ Bio/Jul 6 [E-pub ahead of print]

PFLANZ, R., PETER, A., SCHAFER, U., JACKLE, H. (2004). Follicle separation during Drosophila oogenesis requires the activity of the Kinesin II-associated polypeptide Kap in germline cells. EMBO Reports 5: 510-514.

QIU, P., PAN, PC., GOVIND, S. (1998). A role for the Drosophila Toll/Cactus pathway in larval hematopoiesis. Development 125: 1909-1920.

RUSSELL, SR., SÁNCHEZ-SORIANO, N., WRIGHT, CR., ASHBURNER, M. (1996). The Dichaete gene of Drosophila melanogasterencodes a Sox-domain protein required for embryonic segmentation. Development 122: 3669-3676.

SCHIFFMANN, DA., WHITE, JH., COOPER, A., NUTLEY, MA., HARDING, SE., JUMEL, K., SOLARI, R., RAY, KP., GAY, NJ. (1999). Formation and biochemical characterization of tube/pelle death domain complexes: critical regulators of postreceptor signaling by the Drosophilatoll receptor. Biochemistry38: 11722 11733.

SHELTON, CA., WASSERMAN, SA. (1993). pelle encodes a protein kinase required to establish dorsoventral polarity in the Drosophila embryo. Cel/72: 515-525.

SHEN, B., MANLEY, JL. (1998). Phosphorylation modulates direct interactions between the Toll receptor, Pelle kinase and Tube. Development 125: 47194728.

SHEN, B., MANLEY, JL. (2002). Pelle kinase is activated by autophosphorylation during Toll signaling in Drosophila. Development 1925-1933.

SMITH, JM., KOOPMAN, PA. (2004). The ins and outs of transcriptional control: nucleocytoplasmic shuttling in development and disease. Trends Genet 1: 4-8.

STUKENBERG, PT., LUSTIG, KD., MCGARRY, TJ., KING, RW., KUANG, J., KIRSCHNER, MW. (1997). Systematic identification of mitotic phosphoproteins. Curr Bio/7: 338-348.

TOWB, P., GALINDO, RL., WASSERMAN, SA. (1998). Recruitment of Tube and Pelle to signaling sites at the surface of the Drosophila embryo. Development 125: 2443-2450.

WEGNER, M. (2005). Secrets to a healthy Sox life: lessons for melanocytes. Pigment Cell Res 18: 74-85

WISSMÜLLER, S., KOSIAN, T., WOLF, M., FINZSCH, M., WEGNER, M. (2006). The high-mobility-group domain of Sox proteins interacts with DNA-binding domains of many transcription factors. Nucleic Acids Res 34: 1735-1744.

XIAO, T., TOWB, P., WASSERMAN, SA., SPRANG, SR. (1999). Three-dimensional structure of a complex between the death domains of Pelle and Tube. Cell 99: 545-555.

ZHAO, R., DALEY, GQ. (2008). From fibroblasts to iPS cells: induced pluripotency by defined factors. J Cell Biochem 105: 949-955. 


\section{Further Related Reading, published previously in the Int. J. Dev. Biol.}

See Special Issue Pattern Formation edited by Michael K. Richardson and Cheng-Ming Chuong at: http://www.ijdb.ehu.es/web/contents.php?vol=53\&issue=5-6

Developmental expression of Apnanos during oogenesis and embryogenesis in the parthenogenetic pea aphid Acyrthosiphon pisum

Chun-Che Chang, Ting-Yu Huang, Charles E. Cook, Gee-Way Lin, Chun-Liang Shih and Rita P.-Y. Chen

Int. J. Dev. Biol. (2009) 53: 169-176

Molecular aspects of avian oogenesis and fertilisation

Bozenna Olszanska and Urszula Stepinska

Int. J. Dev. Biol. (2008) 52: 187-194

Accessory nuclei in insect oogenesis: in search of the function of enigmatic organelles Mariusz K. Jaglarz, Malgorzata Kloc and Szczepan M. Bilinski

Int. J. Dev. Biol. (2008) 52: 179-185

Accumulation and dynamics of proteins of the MCM family during mouse oogenesis and the first embryonic cell cycle Lukasz Swiech, Katarzyna Kisiel, Renata Czolowska, Maciej Zientarski and Ewa Borsuk

Int. J. Dev. Biol. (2007) 51: 283-295

Stage-specific regulation of programmed cell death during oogenesis of the medfly Ceratitis capitata (Diptera, Tephritidae) Athanassios D. Velentzas, loannis P. Nezis, Dimitrios J. Stravopodis, Issidora S. Papassideri and Lukas H. Margaritis Int. J. Dev. Biol. (2007) 51: 57-66

Genetic control of dorsoventral patterning and neuroblast specification in the Drosophila Central Nervous System Guoyan Zhao, Scott R. Wheeler and James B. Skeath

Int. J. Dev. Biol. (2007) 51: 107-115

Maternal-effect loci involved in Drosophila oogenesis and embryogenesis: P element-induced mutations on the third chromosome Manolo Bellotto, Daniel Bopp, Kirsten A Senti, Richard Burke, Peter Deak, Peter Maroy, Barry Dickson, Konrad Basler and Ernst Hafen Int. J. Dev. Biol. (2002) 46: 149-157

mgm 1, the earliest sex-specific germline marker in Drosophila, reflects expression of the gene esg in male stem cells Adrian Streit, Luca Bernasconi, Pavel Sergeev, Alex Cruz and Monica Steinmann-Zwicky

Int. J. Dev. Biol. (2002) 46: 159-166

Female sterile mutations and egg chamber development in Drosophila melanogaster S Gigliotti, D Rotoli, A Manzi, F Graziani and C Malva

Int. J. Dev. Biol. (2000) 44: 581-589
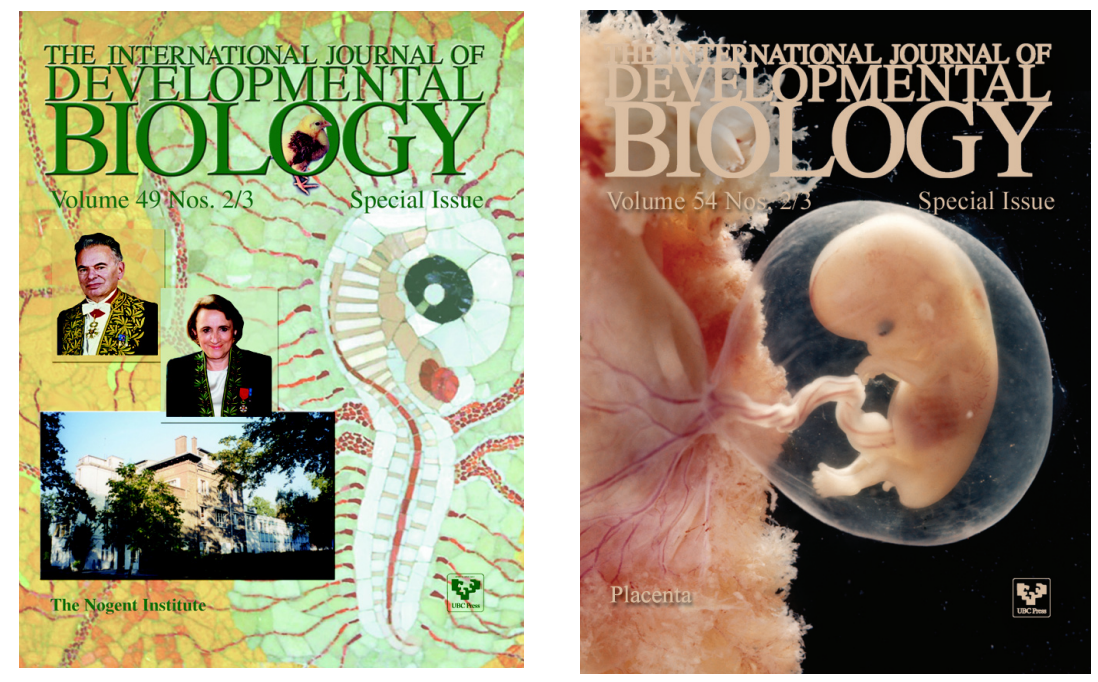

5 yr ISI Impact Factor $(2009)=3.253$
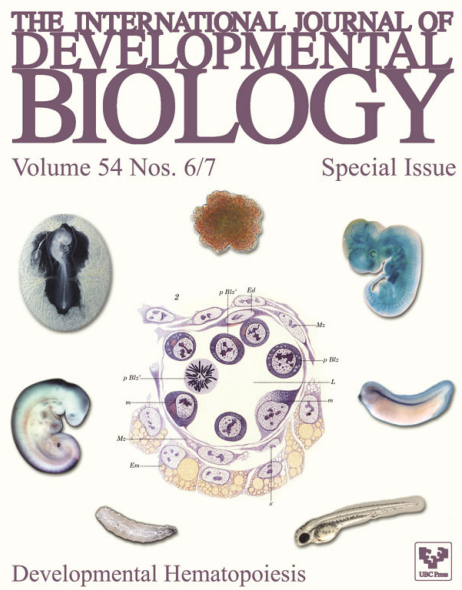

Developmental Hematopoiesis 